\title{
Effect of Primaquine on Gametocyte Carriage in the Case-Management of Uncomplicated Falciparum Malaria with Acts: Nigerian Perspective
}

\author{
A. B. Tsuung1, S. L. Pitmang², F. L. Dassak ${ }^{2}$ \\ ${ }^{1}$ Department of Family Medicine, Yobe State University Teaching Hospital, Damaturu, Yobe State, Nigeria \\ ${ }^{2}$ Department of Family Medicine, Jos University Teaching Hospital, Jos, Plateau State, Nigeria \\ Email: tsuung@yahoo.com,pitmangsl@yahoo.co.uk,felixdassak@yahoo.com
}

How to cite this paper: Tsuung, A.B., Pitmang, S.L. and Dassak, F.L. (2018) Effect of Primaquine on Gametocyte Carriage in the Case-Management of Uncomplicated Falciparum Malaria with Acts: Nigerian Perspective. Advances in Infectious Diseases, 8 , 39-53.

https://doi.org/10.4236/aid.2018.82006

Received: April 8, 2018

Accepted: May 18, 2018

Published: May 21, 2018

Copyright $\odot 2018$ by authors and Scientific Research Publishing Inc. This work is licensed under the Creative Commons Attribution International License (CC BY 4.0). http://creativecommons.org/licenses/by/4.0/

\section{Open Access}

\begin{abstract}
Background: Drugs that kill or inhibit sexual stages of Plasmodium such as Primaqiune (PQ) could potentially amplify or synergize the impact of first line antimalarials by blocking transmission to mosquitoes. This study examined the effect of Primaquine on gametocyte carriage in the case management of uncomplicated falciparum malaria with artemisinin-based combination therapy (ACT) with the overall purpose of possibly recommending it as an adjunct drug for malaria control. Methods: A total of 181 patients with uncomplicated falciparum malaria, normal glucose-6-phosphate dehydrogenase (G6PD) enzyme levels, and haemoglobin levels $\geq 8 \mathrm{~g} / \mathrm{dL}$ completed this two-arm randomized blinded clinical trial to test the efficacy of a single dose PQ $(0.75 \mathrm{mg} / \mathrm{kg})$ on falciparum gametocytaemia. 88 subjects were assigned to a standard 3-day course of Dihydroartemisinin-Piperaquine (DHP) alone ( $\mathrm{n}=$ 88 ) while 93 others had DHP combined with a single dose of PQ on day 3 ( $\mathrm{n}=$ 93). A 28-day follow-up schedule carried out in the outpatient clinic of a Primary health facility in Vom, Plateau State Nigeria where study participants were seen on days 1, 3, 7 and then weekly to assess the presence of asexual parasites and gametocytes by microscopy. A Kaplan-Meier analysis was employed to determine the survival function of gametocytes on day 3. The data was analyzed using Epi info version 7.1.5. Results: With a gametocyte prevalence of $27.1 \%$, gametocyte carriage rate was lower in the PQ group due to higher probability of clearing gametocytes (Breslow test $\chi^{2}=8.306, \mathrm{df}=1, p=$ 0.004 ) and significantly less likely to harbor gametocytes by day 7 when compared to the DHP-alone group $\left(\chi^{2}=6.218, \mathrm{df}=1, p=0.013\right)$. Conclusion: Addition of single-dose $0.75 \mathrm{mg} / \mathrm{kg}$ PQ was associated with reduced gameto-
\end{abstract}


cyte carriage as a result of faster gametocyte clearance and lower incidence of gametocyte development in DHP-treated patients. PQ as gametocytocidal drug may be useful in combination with artemisinin-based combination therapy (ACT) regimen to clear gametocytes and thereby interrupt malaria transmission to mosquito vector more effectively than ACT alone.

\section{Keywords}

Malaria, Artemisinin-Based Combination Therapy, Plasmodium falciparum, Primaquine, Gametocytes, Dihydroartemisinin-Piperaquine

\section{Introduction}

Malaria has remained a global public health challenge for decades [1]. Despite the giant strides taken by World Health Organization (WHO) in reducing the burden of malaria [2], Nigeria and other Sub-Saharan African (SSA) countries are still major contributors to the scourge of this preventable disease [3].

The efforts to reduce the impact of malaria on the population at risk have compelled several malaria endemic nations to implement a number of prevention and control strategies [2] [4]. With research for effective malaria vaccines still ongoing, interventions that reduce vectorial capacity are a necessary first step towards control and possibly eradication [2] [5].

Interestingly, antimalarial treatment failure has been associated with increased gametocyte carriage after treatment implicating gametocytes as early indicators of parasite resistance [6]. Consequently, malaria elimination initiatives and Artemisinin-resistance containment strategies both require additional tools that are specifically aimed at reducing the transmission of malaria parasites [6].

Clinical reports and mathematical models have shown that reduction in the incidence of disease and elimination goals can be achieved with combination therapies that incorporate drugs that not only cure patients but also decrease transmission of Plasmodium falciparum sexual stage parasites to the mosquito vector [7] [8].

The implementation of artemisinin-based combination therapy (ACT) may have beneficial influence on malaria transmission in the general population [5]. However, ACT alone may not be sufficient to completely prevent post-treatment malaria transmission due to persistent post-treatment gametocytaemia [9].

This inadequacy of ACTs prompts the use of Primaquine (PQ) [8], the only drug with proven gametocytocidal activity on mature sexual stage parasite to provide a potent solution to effective malaria treatment, curbing the development of antimalarial resistance and possibly contribute to malarial eradication [10] [11].

There is paucity of data on efficacy of PQ in post-treatment gametocytaemia in ACT treated patients especially in malaria endemic regions where ACTs are recommended first line drugs for malaria treatment [12] [13]. Additionally, 
Primaquine use and its transmission-blocking effect are scarce in Nigeria and SSA where malaria is endemic [13].

The aim of this study was to assess the effect of Primaquine on gametocyte carriage in the treatment of uncomplicated falciparum malaria with Dihyroartemisinin-Piperaquine (DHP) and the gametocyte carriage rate as well as incidence of gametocyte development/clearance time with the overall purpose of possibly recommending it as an adjunct drug for artemisinin-based combination therapy for malaria control. Findings from this study could help inform formulation of national policy where Primaquine, in line with WHO recommendation, may be proffered as an adjunct drug for Artemisinin-based combination therapy for malaria control.

\section{Patients and Methods}

\subsection{Study Design and Area}

A blinded randomized controlled clinical conducted between July 2015 and September 2015 was carried out in the General Outpatient Department of Vom Christian Hospital, Plateau State, Nigeria.

\subsection{Study Population}

This comprised all patients presenting with fever or history of fever within the past 24 hours and microscopically confirmed $P$. falciparum infection regardless of the presence of gametocyte stages attending the general outpatient clinic of the hospital.

\subsection{Study Hypothesis}

The study was devised to test the hypothesis that the addition of a single dose of Primaquine $(0.75 \mathrm{mg} / \mathrm{kg})$ to ACTs for the treatment of uncomplicated falciparum malaria shortens gametocyte clearance time and reduces gametocyte prevalence.

\subsection{Inclusion Criteria}

1) Adults and children aged one year and above or weight $\geq 10 \mathrm{~kg}, 2$ ) Plasmodium parasite density in the range of 1000 asexual parasites per $\mu$ and above, 3) Presence of fever or axillary temperature $\geq 37.5^{\circ} \mathrm{C}$, or positive history of fever in the preceding $24 \mathrm{hrs}$, 4) Normal glucose-6-phosphate dehydrogenase (G6PD) enzyme levels based on a qualitative test, 5) Haemoglobin level of $8 \mathrm{~g} / \mathrm{dL}$ and above, 6) Residence in study area and or ability to come for the stipulated follow-up visits, 7) Ability to take oral medications, 8) Informed consent of participant or parent/guardian of participant.

\subsection{Exclusion Criteria}

1) General danger symptoms and signs of severe malaria; as oral therapy is not indicated in the treatment of severe malaria, 2) Febrile conditions caused by dis- 
eases other than malaria; will not respond to anti-malaria, 3) Allergy to any of the drug components, or had used any of the component drugs within 28 days of enrolment, to avoid complications to therapy and masking effects of previous drug use, 4) Serious concomitant medical illness; or any medical illness not compatible with the study objectives, 5) Pregnancy detected clinically or by urine $\beta$-subunit hCG or lactating mother, drug safety in pregnancy and breastfeeding not ascertained.

All eligible subjects were recruited using a structured questionnaire after training of research assistants. Relevant socio-demographic characteristics (bio-data); medical history, physical examination findings and laboratory investigation were conducted for each patient by the study physician. Physical examination including body temperature was measured using a digital thermometer $\left({ }^{\circ} \mathrm{C}\right)$. The patients were weighed using a reliable standardized weighing scale (Health scale, Techmel \& Techmel, Texas, USA) and recorded to the nearest kilogram, with minimal clothing. The weighing scale was calibrated daily using a known weight. The patients were also examined for pallor, jaundice, splenomegaly and hepatomegaly. The diagnosis of malaria was confirmed by collecting finger prick blood to make both thick and thin film on a slide with $3 \%$ Giemsa stain for 30 minutes [14] by an experienced laboratory scientist who was blinded to the treatment allocations. The thin film was used for identifying the species of malaria parasite while the thick films for parasite count. The parasite density (per uL of blood) was calculated, assuming a normal leucocytes level of 8000 leucocyte/uL using the formula:

$$
\begin{gathered}
\text { Parasite Density }=\frac{(\text { Number of asexual parasite counted } \times \text { WBC count })}{\text { Number of leucocytes counted }} \\
\text { Gametocyte Density }=\frac{(\text { Number of gametocytes } \times \text { WBC count })}{\text { Number of leucocytes counted }}
\end{gathered}
$$

Quality control was done by masked re-reading of $10 \%$ of all slides selected randomly by an independent scientist and was considered negative if no parasite were detected in 200 oil immersion fields. G6PD deficiency was defined using qualitative assays based on the fluorescent spot test (Trinity Biotech, cat no. 203-A) with results classified into three groups normal, intermediate and deficient [15]. The test had a cut-off of approximately $20 \%$ enzyme function, below which there is no function [15]. The WHO classification defines severe G6PD deficiency as $10 \%$ enzyme function [16]. Only participants with a normal test were included in the study.

All consecutively enrolled patients were given an inclusion number and then randomly assigned (in blocks of 12 patients weekly) to receive either a standard 3-day course of DHP (fixed-dose tablets of $40 \mathrm{mg}$ Dihydroartemisinin and 320 mg Piperaquine) with Primaquine (DHP + PQ arm) or without it (DHP-only arm) using computer-generated random number list concealed in sequentially numbered, sealed opaque envelopes and administered (by daily balloting) by the 
study pharmacist. The study pharmacist was the only member of the team not blinded to the treatment arm and was not involved in assessing patient or assigning outcomes. The daily DHP regimen was based on weight ( $\geq 41 \mathrm{~kg}$ : 3 tablets; 31 - $40 \mathrm{~kg}: 2$ tablets; 18 - $30 \mathrm{~kg}: 1$ tablet; 10 - $17 \mathrm{~kg}: 1 / 2$ tablet). The total dose of Dihydroartemisinin ranged $4-10.9 \mathrm{mg} / \mathrm{kg}$, and Piperaquine ranged $32-87.3$ $\mathrm{mg} / \mathrm{kg}$. In the DHP + PQ arm, a single dose of PQ was given on day 3 to achieve a dose of $0.75 \mathrm{mg} / \mathrm{kg}$, rounded to the nearest half tablet. The mean dose was 0.74 $\mathrm{mg} / \mathrm{kg}$ (range, $0.5-0.94 \mathrm{mg} / \mathrm{kg}$ ). The first daily dose for both study groups was administered at the study center supervised by the study physician or an assistant, the second dose the following day by the patient at home and the third (last) dose at the study center. A full drug dose was re-administered if patient spits out or vomits within 30 minutes or half drug dose administered if between 30 mins and one hour. If the patient rejects the drug again, he/she received another anti-malaria drug in accordance with the national guide line but excluded from the study. Patients were instructed on how to administer the doses which fell outside the study time.

Follow up clinical assessment with examination of body temperatures was done on days $0,3,7,14,21$ and 28 or at any time if the patient felt unwell. Subjects with fever $\left(\geq 38.5^{\circ} \mathrm{C}\right.$ using axillary temperature measurement) received Acetaminophen (Paracetamol). Blood sample from finger prick was obtained to make thick and thin smear during each of these follow up days for parasitological examination. Individuals, for whom the treatment failed as defined, were given rescue treatment according to the national guideline and withdrawn from the study [17]. Participants who did not return on schedule for follow up were visited at home same day or phone calls made to remind them of their follow up. All results and outcomes were made available to patients at the end of the study.

The primary outcome measure was the effect of the standard single dose of Primaquine in addition to the completed 3-day treatment with DHP on gametocyte carriage. This was done by comparing the overall risk of gametocyte carriages on day 7 and then weekly till day 28 , to measure the combined effect on gametocyte development and clearance (primary endpoint).

This two-arm trial required 84 patients per arm to detect a 7\% absolute reduction in the prevalence of gametocytes following the week after treatment with PQ from $9 \%$ in the DHP-only arm to $2 \%$ in the DHP + PQ arm with $80 \%$ power and a 2 -sided $\alpha$ of 0.05 . The anticipated gametocyte prevalence of $9 \%$ was based on the day 7 prevalence in earlier studies with DHP [18].

The impact of PQ on gametocytes was assessed using the following secondary endpoints: 1) The gametocyte clearance rates by day 28 in patients with gametocytes on day 3,2 ) The incidence of gametocyte development by day 28 in patients who were gametocyte-free on day 3,3) Gametocyte densities between days 3 and 28 inclusive.

Data generated were analyzed using Epi-info 7.1.5 version, Centre for Disease Control and Prevention Atlanta Georgia software for computer analysis [19]. Quantitative and qualitative data were expressed as mean with standard devia- 
tion (SD) and number of observations with percentages (\%), respectively. All $\mathrm{P}$-values reported were from two-tailed testing, and statistical significance was set at 0.05 . Descriptive statistics were used to summarize baseline values and demographic data. The chi-square or Fisher's exact tests were used to compare proportions as appropriate, and the $t$-test was used to analyze continuous data. The primary analysis (effect of PQ on gametocyte carriage) was based on a modified intent-to-treat analysis, while differences in gametocyte prevalence were also compared using $t$-test along with corresponding $\mathrm{P}$ values. Unadjusted Kaplan-Meier survival analysis was used to compare the cumulative risk of gametocyte development in the gametocyte-free population on day 3 , and to measure the risk of persistence of gametocytes or appearance of new gametocytes in those gametocytemic on day 3 .

\section{Results}

A total of 181 patients (88 DHP-only arm and 93 DHP + PQ arm) were studied (Figure 1).

\subsection{Baseline Characteristics}

Table 1 showed that randomization was effective as there were no significant differences in the parameters in the groups.

\subsection{Parasite Count}

\section{Overview of Mean Parasite Count on Different Days}

The mean and standard deviation of the parasite count at baseline between the treatment arms revealed that there was no significant difference $(p=0.402)$. There was significant difference in mean parasite count between the two treatment arms at day $3(\mathrm{t}=2.912, p=0.004)$, and there was a significant mean difference in parasite count on day $7(\mathrm{t}=5.380, p=0.0005)$; day $14(\mathrm{t}=6.690, p=$ $0.0005)$. Table 2 shows the results summary.

\subsection{Gametocyte Responses}

The gametocyte count was found in the peripheral blood of a total of 49 patients (27.1\%); and results revealed that there was no significant mean difference in gametocyte count at baseline between treatment arms $(p=0.658)$; and also, there was no significant mean difference in gametocyte count after 72 hours $(p=$ $0.407)$ and day $7(p=0.120)$. Table 3 shows the result summary.

\subsubsection{Gametocyte Clearance Time (GCT)}

In the DHP treatment arm, 9 study subjects completely cleared gametocytes within 72 hours, while 6 subjects cleared in 168 hours. Therefore,

$$
\begin{aligned}
& \text { Gametocyte clearance time }(\text { GCT })_{\text {DHP }} \\
& =\frac{(9 \times 72)+(11 \times 168)+(3 \times 336)}{23}=\frac{1656}{23}=152.3 \text { hours }
\end{aligned}
$$




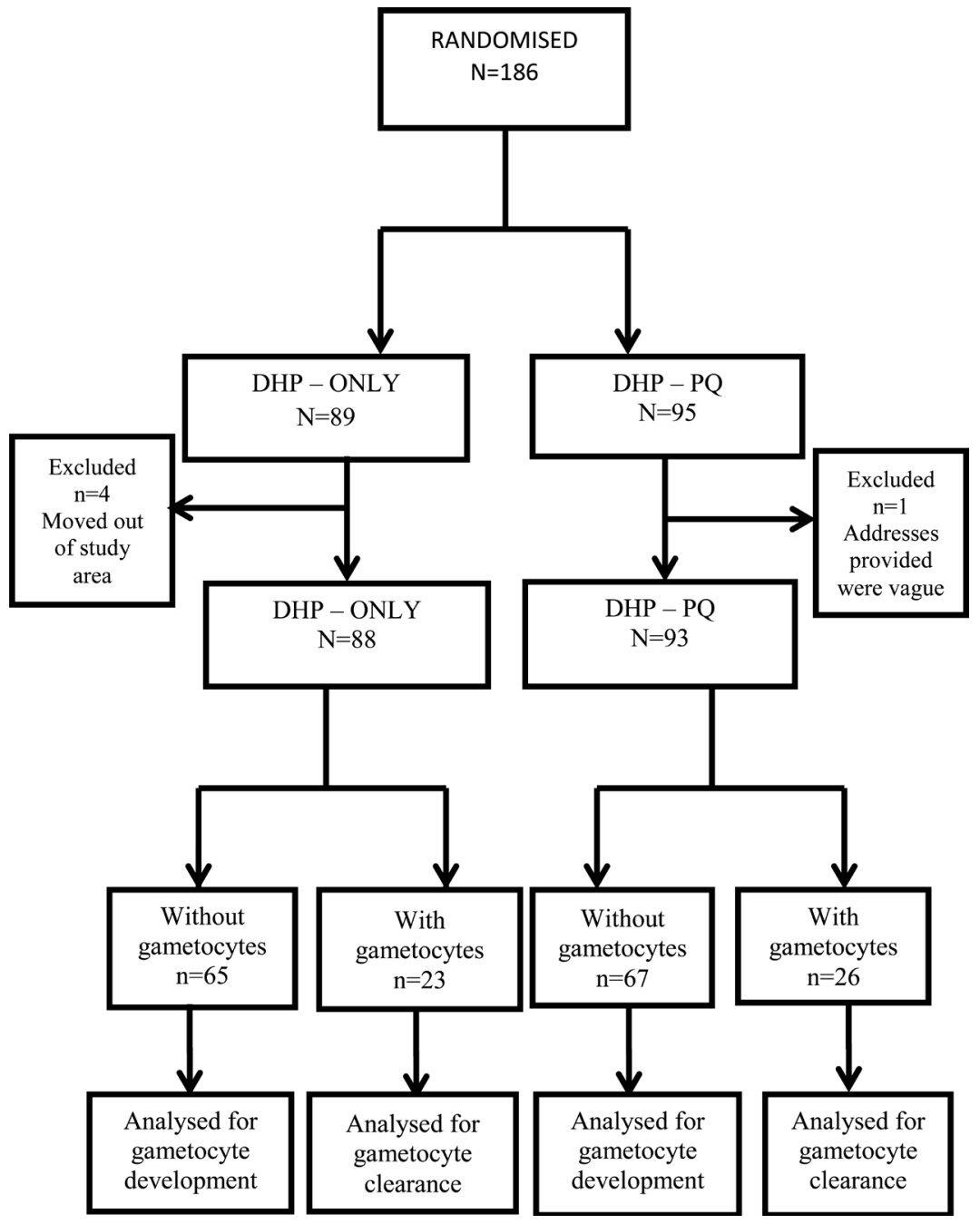

Figure 1. Study profile.

Table 1. Baseline characteristics.

\begin{tabular}{|c|c|c|c|c|}
\hline & \multicolumn{2}{|c|}{ Drug Treatment Arm } & \multirow{2}{*}{$\begin{array}{l}\text { Chi-square/ } \\
\text { t-test value }\end{array}$} & \multirow[b]{2}{*}{ P-value } \\
\hline & $\begin{array}{c}\text { DHP } \\
(\mathrm{n}=87)\end{array}$ & $\begin{array}{c}\mathrm{DHP}+\mathrm{PQ} \\
(\mathrm{n}=93)\end{array}$ & & \\
\hline Mean \pm SD age (Years) & $32.48 \pm 14.49$ & $35.77 \pm 14.50$ & 1.530 & $0.128^{\ddagger}$ \\
\hline $\begin{array}{c}\text { Adults } \\
\text { Children (age }>\text { I year) }\end{array}$ & $\begin{array}{c}69 \\
8\end{array}$ & $\begin{array}{c}80 \\
6\end{array}$ & 10.211 & $0.511^{\#}$ \\
\hline $\begin{array}{c}\text { Male } \\
\text { Female }\end{array}$ & $\begin{array}{l}26 \\
30\end{array}$ & $\begin{array}{l}62 \\
63\end{array}$ & 0.156 & $0.693^{\#}$ \\
\hline Mean \pm SD weight $(\mathrm{kg})$ & $59.91 \pm 18.35$ & $61.60 \pm 16.36$ & 0.656 & $0.513^{\ddagger}$ \\
\hline Mean $\pm \mathrm{SD}$ temperature $\left({ }^{\circ} \mathrm{C}\right)$ & $37.54 \pm 0.89$ & $37.60 \pm 0.65$ & 0.591 & $0.555^{\ddagger}$ \\
\hline Range of parasitemia (count/L) & $1000-1980$ & $1170-1996$ & & \\
\hline Mean \pm SD (parasite count) $(u / l)$ & $1437.31 \pm 190.50$ & $1460.62 \pm 183.10$ & 0.840 & $0.402^{\ddagger}$ \\
\hline Mean \pm SD (gametocyte count) $(u / l)$ & $16.02 \pm 30.50$ & $20.32 \pm 28$ & 0.983 & $0.327^{\dagger}$ \\
\hline Haemoglobin Estimation (\%) & $16.02 \pm 30.50$ & $14.19 \pm 24.86$ & 0.548 & $0.584^{\ddagger}$ \\
\hline
\end{tabular}

${ }^{\dagger}$ Independent t-test; ${ }^{\#}$ Chi-square. 
Table 2. Overview of mean parasite count on different follow up days.

\begin{tabular}{ccccc}
\hline Day & Treatment Arm & Mean & t-test & p-value \\
\hline \multirow{1}{*}{$\mathbf{1}$} & DHP & $1437.31 \pm 190.50$ & 0.840 & 0.402 \\
& DHP + PQ & $1460.62 \pm 183.10$ & & \\
$\mathbf{3}$ & DHP & $713.57 \pm 168.57$ & 2.912 & 0.004 \\
& DHP + PQ & $634.04 \pm 196.87$ & & \\
7 & DHP & $298.58 \pm 135.66$ & 5.380 & 0.0005 \\
\multirow{2}{*}{$\mathbf{1 4}$} & DHP + PQ & $186.29 \pm 144.66$ & & \\
& DHP & $26.44 \pm 29.18$ & 6.690 & 0.0005 \\
\hline
\end{tabular}

Table 3. Overview of mean gametocyte count.

\begin{tabular}{cccccc}
\hline Day & Treatment Arm & N & Mean & t-test & p-value \\
\hline \multirow{1}{*}{$\mathbf{1}$} & DHP & 23 & $16.02 \pm 30.50$ & & \\
& DHP + PQ & 26 & $14.19 \pm 24.86$ & 0.443 & 0.658 \\
3 & DHP & 14 & $5.28 \pm 12.67$ & & \\
& DHP + PQ & 12 & $3.87 \pm 10.11$ & 0.832 & 0.407 \\
7 & DHP & 3 & $0.28 \pm 1.76$ & & \\
& DHP + PQ & 0 & $0.00 \pm 0.00$ & 1.562 & 0.120 \\
\hline
\end{tabular}

In the DHP + PQ treatment arm, 14 study subject completely cleared gametocytes within 72 hours, while 12 subjects cleared in 168 hours. Therefore,

$$
\begin{aligned}
& \text { Gametocyte clearance time }(\mathrm{GCT})_{\mathrm{DHP}+\mathrm{PQ}} \\
& =\frac{(14 \times 72)+(12 \times 168)}{26}=\frac{648}{26}=116.3 \text { hours }
\end{aligned}
$$

The gametocyte clearance time in the DHP + PQ treatment arm (116.3 hours) was faster than that of the DHP treatment arm (152.3 hours) $p=0.589$. Figure 2 shows the mean gametocyte count for follow-up days.

\subsubsection{Rate of Gametocyte Clearance}

The Kaplan-Meier test was used to determine the rate of gametocyte clearance. The time of visit was measured in time intervals of 28 days in between visits. The Kaplan-Meier chart (Figure 3) shows that participants in the DHP + PQ arm had significantly higher probability of clearing gametocytes than the DHP treatment group within the earlier time of the study (Breslow test: $\chi^{2}=8.306, \mathrm{df}$ $=1, p=0.004)$. Also, after day 7 , the result revealed that participants in the DHP study arm had a significantly higher probability of having/developing gametocytes than the DHP + PQ arm (Tarone-Ware: $\chi^{2}=7.310$, $\left.\mathrm{df}=1, p=0.007\right)$. At the end of the time frame, the result showed that the study groups were significantly different in the time it took to clear gametocytes. The Mantel Cox test revealed that the control group had a higher probability of having gametocytes than the DHP + PQ group $\left(\chi^{2}=6.218, \mathrm{df}=1, p=0.013\right)$. 


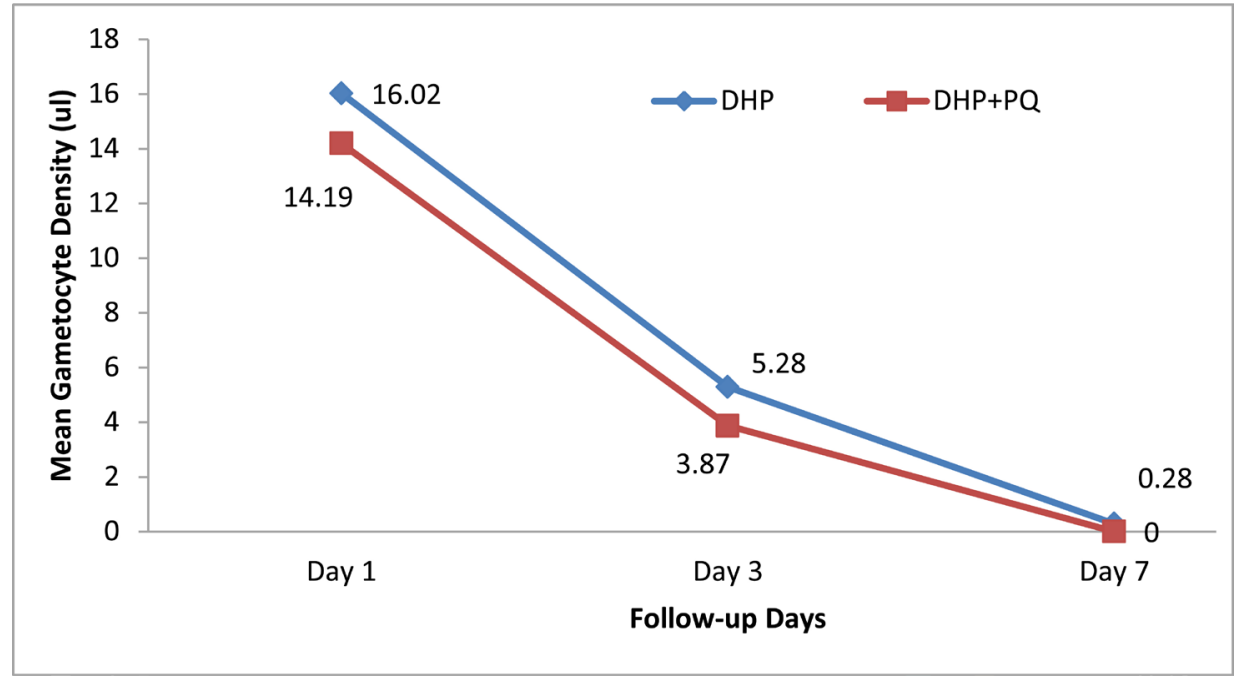

Figure 2. Mean gametocytes density on follow up days.

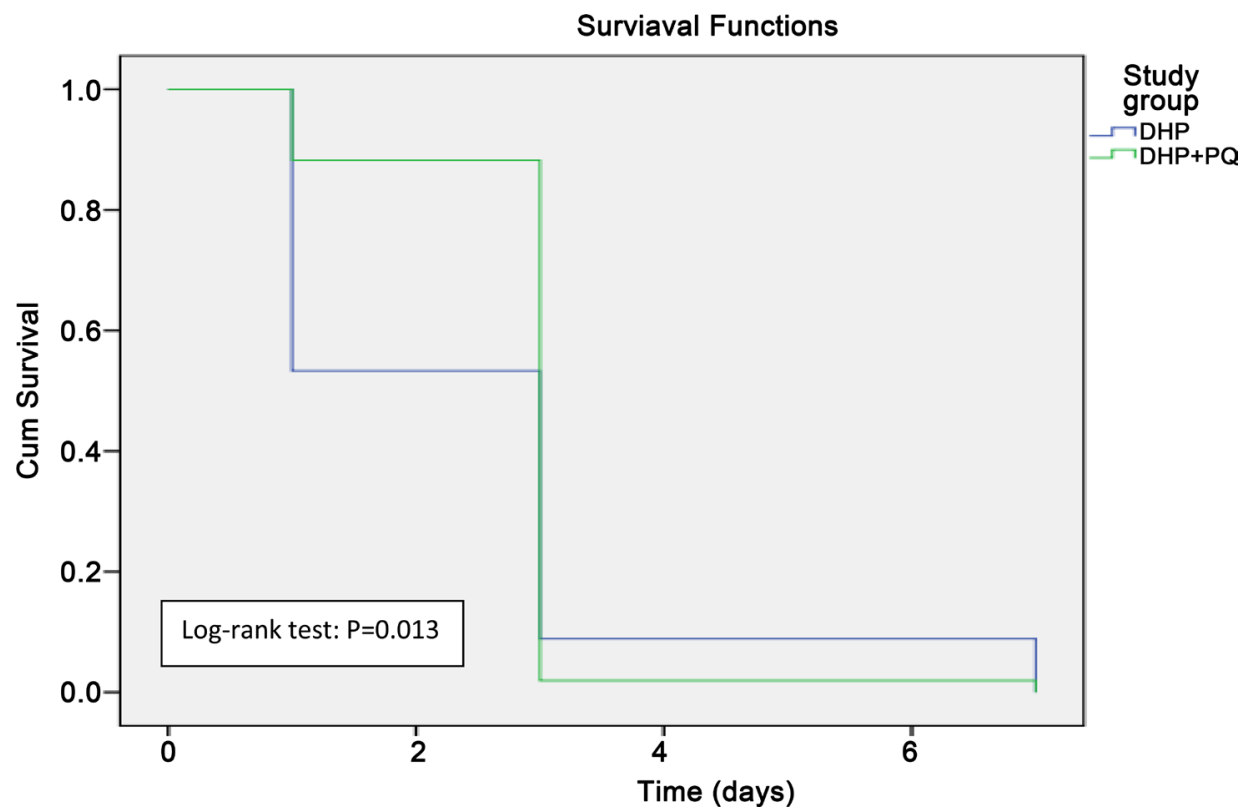

Figure 3. Kaplan-Meier chart for rate of gametocyte clearance between the study groups.

\subsection{Haemoglobin Changes}

Results revealed that there was no significant mean haemoglobin difference between the DHP and DHP + PQ treatment arms respectively $(p=0.584)$; there was no significant mean haemoglobin difference between the treatment arms in day $3(p=0.783)$; also, there was no significant mean difference in day 7 ( $p=$ $0.639)$; day $14(p=0.724)$. Furthermore, there was no significant mean difference in haemoglobin between the treatment arms in day $21(p=0.842)$, and in day $28(p=0.318)$. Table 4 shows the summary of the result.

\section{Discussion}

This study showed that gametocyte prevalence at enrolment was $27.1 \%$. This is 
Table 4. Comparison of mean haemoglobin differences between the study groups.

\begin{tabular}{|c|c|c|c|c|}
\hline Day & Treatment Arm & Mean \pm SD Haemoglobin (\%) & t-test & p-value \\
\hline \multirow{2}{*}{1} & DHP & $13.92 \pm 1.78$ & \multirow{2}{*}{0.644} & \multirow{2}{*}{0.584} \\
\hline & $\mathrm{DHP}+\mathrm{PQ}$ & $14.07 \pm 1.89$ & & \\
\hline \multirow{2}{*}{3} & DHP & $13.87 \pm 1.71$ & \multirow{2}{*}{0.276} & \multirow{2}{*}{0.783} \\
\hline & $\mathrm{DHP}+\mathrm{PQ}$ & $13.94 \pm 1.70$ & & \\
\hline \multirow{2}{*}{7} & DHP & $13.89 \pm 1.60$ & \multirow{2}{*}{0.470} & \multirow{2}{*}{0.639} \\
\hline & $\mathrm{DHP}+\mathrm{PQ}$ & $14.01 \pm 1.68$ & & \\
\hline \multirow{2}{*}{14} & DHP & $14.02 \pm 1.58$ & \multirow{2}{*}{0.353} & \multirow{2}{*}{0.724} \\
\hline & $\mathrm{DHP}+\mathrm{PQ}$ & $13.93 \pm 1.67$ & & \\
\hline \multirow{2}{*}{21} & DHP & $13.96 \pm 1.62$ & \multirow{2}{*}{0.200} & \multirow{2}{*}{0.842} \\
\hline & $\mathrm{DHP}+\mathrm{PQ}$ & $14.01 \pm 1.63$ & & \\
\hline \multirow{2}{*}{28} & DHP & $14.23 \pm 1.71$ & \multirow{2}{*}{1.001} & \multirow{2}{*}{0.318} \\
\hline & $\mathrm{DHP}+\mathrm{PQ}$ & $14.47 \pm 1.51$ & & \\
\hline
\end{tabular}

higher than the $25.5 \%$ prevalence documented by Oguche and colleagues in a study in Nigerian children published in 2014 [20]. Their slightly lower prevalence might be because they reported the national average from different regions rather than a single region done by this study. In a study in Kenya, Bousema et al. also recorded a lower overall prevalence by microscopy of $22.9 \%$ [8]. The variation attributed to user dependency of microscopy might be a reason. Smithuis et al. however, reported an average of $32.8 \%$ in large study in Myanmar which was well higher than this study [21]. The use of molecular detection methods such as PCR employed in their study may account for the higher result.

The mean duration of gametocyte carriage in the study participants was seven days. A similar result was reported by Eziefula et al. where a mean duration of 6.6 days in the group with $0.75 \mathrm{mg} / \mathrm{kg}$ PQ was observed [12]. Tangpukdee and colleagues found a mean duration of 6.4 days [22] [23]. The fact that these studies all looked at the gametocyte responses particularly in the Plasmodium falciparum species that is predominant in the study participants might be a probable explanation for these similarities in duration.

Gametocyte clearance time (GCT) was the time from the initiation of treatment until the first time a slide was gametocyte negative, and remained so for the next 24 hours. The mean gametocyte clearance time was 163 hours without intervention (e.g. PQ) [24]. The GCT observed in the control group of this study was shorter (152.3 hours) probably due to the variation of the effects of ACT regimen on gametocytes. Furthermore, a faster gametocyte clearance time was found in the intervention group that had Primaquine. Thus, patients with gametocytes on day 3 cleared gametocytes faster as shown by the gametocyte clearance times: intervention group (DHP + PQ) (116.3 hours) versus control group (DHP-only) (152.3 hours). This finding compares to studies by Piyaphanee, Tangpukdee and Eziefula most likely due to their comparable ACT regimen, baseline study populations and study protocols [12] [22] [23].

The survival analysis also showed that participants in the DHP + PQ arm had 
significantly lower probability of having/clearing gametocytes than the DHP treatment group during the study period $(\mathrm{p}=0.004)$. In addition, between the day 3 and day 28, study results revealed that participants in the DHP study arm had a significantly higher probability of having/clearing gametocytes than the $\mathrm{DHP}+\mathrm{PQ}$ arm $(\mathrm{p}=0.007)$. This finding agrees with that observed by Sutanto and colleagues [18]. This may likely be explained by the similar duration of follow up of 28 days employed by their study as well as similarities in the doses of PQ used.

ACTs have been shown to have benefits beyond parasitologic and clinical cure but also proved from studies to reduce malaria transmission to the mosquito vector [25]. However, their effects are limited to immature gametocytes predisposing to post-treatment gametocytaemia due to mature gametocytes [8] [21]. This persistent gametocyte carriage rate despite use of ACTs is supported by the finding of this study showing clearance of asexual parasites in both study groups except detectable gametocytes by microscopy until day 7 in only the DHP-only arm.

This result is consistent with previous reports by Tangpukdee et al. in symptomatic malaria patients treated with Artemisinins or ACTs showing persistent mature blood-stage gametocytemia that were not affected by the Artemisinins/ACTs [22] [23]. Similarly, Smithuis and colleagues studied the efficacy of the four recommended ACT regimen in current use against falciparum malaria and found that there was persistent gametocytaemia after ACT which correlated to recurrent infections (recrudescence and re-infections) [21]. Another study showed that ACTs had moderate effects on gametocytes resulting in persistent gametocytaemia after treatment with ACT especially in areas with high malaria transmission [8]. As ACTs are the first-line drugs for malaria with excellent therapeutic profile acknowledged globally, these similarities in the mentioned studies may be related to the predominant antimalarial used (ACT) making it unlikely for studies to employ other drugs for comparison.

While PQ has no effects on asexual parasites, it has synergistic activity with ACTs [26] [27]. ACTs clears asexual parasites preventing gametocyte development and has gametocytocidal action on immature developing gametocytes (by their activity against precursors of this sexual stage) [8]. This is seen in this study by the absence of gametocytes in patients without gametocytes from the baseline to day 28 of the follow up period. Studies by Sutanto and colleagues had a similar pattern as none of the patients among the controls that were gametocyte-free developed microscopically patent gametocytes during the study period [18].

A likely reason for this similarity may be that they also used microcopy for assessment of gametocyte in their study. PQ on the other hand, has gametocytocidal effects on the mature gametocytes and accelerates their clearance from the circulation [16] [28]. This study showed that by day 7, gametocytes were not detected in all patients $(0 \%)$ taking DHP + PQ compared to $13 \%$ (3 patients) taking DHP alone. This was similar to the findings of Wilairatana and Tangpukdee 
while studying the effect of PQ on Plasmodium falciparum transmission [22] [23]. They also found gametocytaemia in $13.1 \%$ of patients that took only ACT on day 7. As mentioned earlier, use of microscopy for detection and quantification of gametocytes in their study is a probable reason for the similarity. Smithuis et al. showed that addition of a single dose of PQ $(0.75 \mathrm{mg}$ base $/ \mathrm{kg})$ given to falciparum malaria with ACT treatment substantially accelerated gametocyte clearance [21]. More so, Tangpukdee also found that adding PQ in ACT may be more effective in eradication of gametocyte than ACT alone [23]. Bousema et al. showed that the potential of $P$. falciparum transmission remains high even after treatment with ACTs although the prevalence and density of gametocytes is lower after Artesunate and SP treatment in Kenya patients [8].

On the contrary, studies from Sudan and India showed reduced or no effect of PQ [29] [30]. These studies agree with Okell et al., who in a randomized controlled study also showed conclusively that a six-dose course of Artemether-Lumefantrine (co-artemether) given to children with $P$. falciparum malaria in Gambia reduces gametocyte prevalence, duration of gametocyte carriage, and infectiousness to mosquitoes [31]. They hypothesized that ACTs sufficiently cleared both asexual and sexual parasites (gametocytes). A possible explanation might be the doses of PQ used in such studies. PQ given in doses less than $0.4 \mathrm{mg} / \mathrm{kg}$ produced equivocal results when malaria transmission was the primary outcome measure [25].

\subsection{Limitations of the Study}

The short duration of the study could not allow conclusive assessment of the effect of gametocytes on recrudescent infections as well as reinfections. Additionally, most studies attest to the considerable contribution of sub-microscopic gametocytaemia to mosquito infection [30] [32]. Measuring the effects of the different treatments on submicroscopic gametocytaemia using molecular methods of gametocyte detection such as the PCR would have increased the power of the study since microscopy notoriously underestimates gametocytes counts [30]. However, microscopy may provide a basis for further studies to build upon especially in developing nations.

\subsection{Conclusion}

The addition of a single dose of PQ could thus have a major effect on malaria transmission from treated patients by shortening the period of infectivity to mosquito vector without compromising efficacy and safety as demonstrated in the study. PQ added to the current first-line ACTs rapidly reduced microscopically patent gametocytaemia within a week of administration with minimum reportage of adverse events as well as insignificant changes in haemoglobin concentration between the study groups.

\subsection{Recommendations}

This study contributes to the fight against malaria in a two-pronged approach: 
by providing research on the effect of antimalarials in disrupting malaria transmission from vectors to humans as well as prevention of the development of antimalarial drug resistance partly attributed to persistent gametocyte carriage.

Further studies of longer duration using real-time PCR assays for gametocyte counts including mosquito feeding studies needs to be conducted to fully elucidate the transmission blocking activity of PQ. In addition, larger studies involving asymptomatic patients should be done to evaluate the efficacy, tolerability and appropriate dose of PQ especially in areas where G6PD testing is not widely available. These will add to the existing evidence for advocacy of policy recommendation of $\mathrm{PQ}$ as an adjunct to existing $\mathrm{ACT}$ regimen for a durable malaria control in Nigeria.

\subsection{Declaration}

Ethical approval for the study was obtained from the Jos University Teaching Hospital ethical committee and permission from the management of the Vom Christian Hospital. Funding for the research was provided by the authors. There is no conflict of interest as the authors have no financial or personal relationships that may have influenced this study.

\section{Acknowledgements}

The authors thank Professor S. Manu for editorial assistance with this article. We remain grateful to Chundusu Daniel, Yusuf Bulus and Noro Samson for their selfless laboratory support.

\section{References}

[1] Johansson, E., Cibulskis, R. and Steketee, R. (2010) Global Partnership to Roll Back Malaria. Malaria Funding and Resource Utilization: The First Decade of Roll Back Malaria. World Health Organization, Geneva, Switzerland.

[2] World Health Organization (2014) Global Malaria Control and Elimination: Report of a Technical Review 2008. World Health Organization, Geneva. http://www.who.int/malaria/publications/atoz/9789241596756

[3] (2013) World Health Organization: World Malaria Report 2013. World Health Organization, Geneva.

http://www.who.int/malaria/publications/world_malaria_report_2013/wmr2013_no _profiles.pdf?ua $=1$

[4] Murray, C.J., Rosenfeld, L.C., Lim, S.S., Andrews, K.G., Foreman, K.J., Haring, D., et al. (2012) Global Malaria Mortality between 1980 and 2010: A Systematic Analysis. Lancet, 379, 413-431. https://doi.org/10.1016/S0140-6736(12)60034-8

[5] Moonen, B., Cohen, J.M., Snow, R.W., Slutsker, L., Drakeley, C., Smith, D.L., et al. (2010) Operational Strategies to Achieve and Maintain Malaria Elimination. Lancet, 376, 1592-1603. https://doi.org/10.1016/S0140-6736(10)61269-X

[6] White, N.J. (2010) Artemisinin Resistance: The Clock Is Ticking. Lancet, 376, 2051-2056. https://doi.org/10.1016/S0140-6736(10)61963-0

[7] Bousema, T. and Drakeley, C. (2011) Epidemiology and Infectivity of Plasmodium falciparum and Plasmodium vivax Gametocytes in Relation to Malaria Control and 
Elimination. Clin Microbiol Rev., 24, 377-410.

[8] Bousema, J.T., Schneider, P., Gouana, L.C., Drakeley, C.J., Tostmann, A., Houben, R., et al. (2006) Moderate Effect of Artemisinin-Based Combination Therapy on Transmission of Plasmodium falciparum. Journal of Infectious Diseases, 193, 1151-1159. https://doi.org/10.1086/503051

[9] Graves, P.M., Gelband, H. and Garner, P. (2012) Primaquine for Reducing Plasmodium falciparum Transmission. Cochrane Database of Systematic Reviews, 9 , CD008152.

[10] Roberts, L. and Malaria, E.M. (2007) Did They Really Say ... Eradication? Science, 318, 1544-1545. https://doi.org/10.1126/science.318.5856.1544

[11] Sinclair, D., Zani, B., Donegan, S., Olliaro, P. and Garner, P. (2009) Artemisinin-Based Combination Therapy for Treating Uncomplicated Malaria. Cochrane Database of Systematic Reviews, No. 3, Art. No.: CD007483. https://doi.org/10.1002/14651858.CD007483

[12] Eziefula, A.C., Gosling, R. and Hwang, J. (2012) Rationale for Short Course Primaquine in Africa to Interrupt Malaria Transmission. Malaria Journal, 11, 360. https://doi.org/10.1186/1475-2875-11-360

[13] White, N.J., Qiao, L.G. and Qi, G. (2012) Rationale for Recommending a Lower Dose of Primaquine as a Plasmodium falciparum Gametocytocide in Populations Where G6PD Deficiency Is Common. Malaria Journal, 11, 418.

https://doi.org/10.1186/1475-2875-11-418

[14] Adu-Gyasi, D., Adams, M., Amoako, S., Mohama, E., Nsoh, M., Amenga-Etego, S., et al. (2012) Estimating Malaria Parasite Density: Assumed White Blood Cell Count of 10,000/ul of Blood Is Appropriate Measure in Central Ghana. Malaria Journal, 10, 238. https://doi.org/10.1186/1475-2875-11-238

[15] Howes, R.E., Battle, K.E. and Satyagraha, A.W. (2013) G6PD Deficiency: Global Distribution, Genetic Variants and Primaquine Therapy. Advances in Parasitology, 81, 133-201. https://doi.org/10.1016/B978-0-12-407826-0.00004-7

[16] World Health Organization Global Malaria Programme: Updated WHO Policy Recommendation (2012) Single Dose Primaquine as a Gametocytocide in Plasmodium falciparum Malaria. http://www.who.int/malaria/publications/atoz/who_pq_policy_recommendation/en $\underline{1}$

[17] WHO (2010) Guidelines for the Treatment of Malaria. 2nd Edition, World Health Organization, Geneva.

[18] Sutanto, I., Suprijanto, S., Kosasih, A., Dahlan, M.S., Syafruddin, D., Kusriastutiet, R., et al. (2013) The Effect of Primaquine on Gametocyte Development and Clearance in the Treatment of Uncomplicated Falciparum Malaria with Dihydroartemisinin Piperaquine in South Sumatra, Western Indonesia: An Open-Label, Randomized, Controlled Trial. Clinical Infectious Diseases, 56, 685-693. https://doi.org/10.1093/cid/cis959

[19] Epi-Info Version 7.1.5. Centre for Disease Control and Prevention. Atlanta Georgia, USA. https://www.cdc.gov/epiinfo/support/downloads/prevversions.html

[20] Oguche, S., Okafor, H.U., Watila, I., Meremikwu, M., Agomo, P., Ogala, W., et al. (2014) Efficacy of Artemisinin-Based Combination Treatments of Uncomplicated Falciparum Malaria in Under-Five-Year-Old Nigerian Children. The American Journal of Tropical Medicine and Hygiene, 91, 925-935. https://doi.org/10.4269/ajtmh.13-0248

[21] Smithuis, F. (2010) Effectiveness of Five Artemisinin Combination Regimens with 
or without Primaquine in Uncomplicated Falciparum Malaria: An Open-Label Randomised Trial. The Lancet Infectious Diseases, 10, 673-681. https://doi.org/10.1016/S1473-3099(10)70187-0

[22] Piyaphanee, W., Krudsood, S., Tangpukdee, N., Thanachartwet, W., Silachamroon, U., Phophak, N., et al. (2006) Emergence and Clearance of Gametocytes in Uncomplicated Plasmodium Falciparum Malaria. The American Journal of Tropical Medicine and Hygiene, 74, 432-435.

[23] Tangpukdee, N., Krudsood, S., Srivilairit, S., Phophak, N., Chonsawat, P., Yanpanich, W., et al. (2008) Gametocyte Clearance in Uncomplicated and Severe Plasmodium falciparum Malaria after Artesunate-Mefloquine Treatment in Thailand. The Korean Journal of Parasitology, 46, 65-70.

https://doi.org/10.3347/kjp.2008.46.2.65

[24] Lawpoolsri, S., Klein, E.Y. and Singhasivanon, P. (2009) Optimally Timing Primaquine Treatment to Reduce Plasmodium falciparum Transmission in Low Endemicity Thai-Myanmar Border Populations. Malaria Journal, 8, 159.

https://doi.org/10.1186/1475-2875-8-159

[25] Garner, P. and Graves, P.M. (2005) The Benefits of Artemisinin Combination Therapy for Malaria Extend beyond the Individual Patient. PLoS Med, 2, e105. https://doi.org/10.1371/journal.pmed.0020105

[26] Dondorp, A.M., Nosten, F., Yi, P., Das, D., Phyo, A.P. and Tarning, J. (2009) Artemisinin Resistance in Plasmodium falciparum Malaria. The New England Journal of Medicine, 361, 455-467. https://doi.org/10.1056/NEJMoa0808859

[27] Phyo, A.P., Nkhoma, S., Ashley, E., Nair, S., McGready, R., Moo, C.L., et al. (2012) Emergence of Artemisinin Resistant Malaria on the Western Border of Thailand. The Lancet, 379, 1960-1966. https://doi.org/10.1016/S0140-6736(12)60484-X

[28] Maeseneer, J.D. and Flinkenflögel, M. (2010) Primary Health Care in Africa: Do Family Physicians Fit in? British Journal of General Practice, 60, 286-292. https://doi.org/10.3399/bjgp10X483977

[29] Kamtekar, K.D. (2004) A Prospective Study Evaluating the Efficacy of a Single, 45-mg Dose of Primaquine, as a Gametocytocidal Agent, in Patients with Plasmodium falciparum Malaria in Mumbai, India. Annals of Tropical Medicine and Parasitology, 98, 453-458. https://doi.org/10.1179/000349804225003550

[30] El-Sayed, B., El-Zaki, S.E., Babiker, H., Gadalla, N., Ageep, T., Mansour, F., Baraka, O., Milligan, P. and Babiker, A. (2007) A Randomized Open-Label Trial of Artesunate-Sulfadoxine-Pyrimethamine with or without Primaquine for Elimination of Sub-Microscopic P. falciparum Parasitaemia and Gametocyte Carriage in Eastern Sudan. PLOS ONE, 2, e1311. https://doi.org/10.1371/journal.pone.0001311

[31] Okell, L.C., Drakeley, C.J., Ghani, A.C., Bousema, T. and Sutherland, C.J. (2008) Reduction of Transmission from Malaria Patients by Artemisinin Combination Therapies: A Pooled Analysis of Six Randomized Trials. Malaria Journal, 7, e125. https://doi.org/10.1186/1475-2875-7-125

[32] Shekalaghe, S., Drakeley, C., Gosling, R., Ndaro, A., van Meegeren, M., Enevold, A., Alifrangis, M., Mosha, F., Sauerwein, R. and Bousema, T. (2007) Primaquine Clears Submicroscopic Plasmodium falciparum Gametocytes That Persist after Treatment with Sulphadoxine-Pyrimethamine and Artesunate. PLOS ONE, 2, e1023. https://doi.org/10.1371/journal.pone.0001023 